\title{
FERMA: An Efficient Geocasting Protocol for Wireless Sensor Networks with Multiple Target Regions ${ }^{\star}$
}

\author{
Young-Mi Song, Sung-Hee Lee, and Young-Bae Ko \\ College of Information and Communication, \\ Ajou University, Suwon, South Korea \\ \{ymsong, sunghee, youngko\}@ajou.ac.kr
}

\begin{abstract}
Some sensor applications are interested in collecting data from multiple regions. For supporting such applications with multiple target regions, most conventional protocols are based on either a network flooding or multiple unicastig to cover those more than one target region. Either one will result in a lot of redundant packets to transmit by energy scared sensor nodes. To alleviate this problem, we propose a novel geocasting scheme which can make a suitable shared path among multiple target regions. We utilize the theorem of "Fermat Point," in order to find an optimal junction point branching into each region. By using this shared path, an interest dissemination can be performed very efficiently. Our simulation study shows that the proposed scheme FERMA reduces a lot of network traffic and achieves significant energy saving as the number of target regions increase.
\end{abstract}

\section{Introduction}

Advances in wireless embedded technologies make it possible to enable small and resource-limited sensor devices to have wireless communication and computational capabilities [1]. Wireless sensor networks (WSNs) with a large number of such smart sensors can be deployed for tracking targets or gathering information about physical phenomena. For many applications in wireless sensor networks [2, 3], a query (also, called as an interest) is commonly used to have sensor nodes collect data from their environments and return these sensing data to a query initiator (i.e., the originator of the interest message). In such query-based sensor networks, an interest message specifies a particular condition to match events; for example, a type of sensing tasks, location information where interesting events might occur, an interval between data propagations, and the lifetime of the query.

There are several approaches for efficiently disseminating interest messages to a target region. A flooding mechanism, which requires any intermediate receiver to rebroadcast a non-duplicated interest packet to all its neighbors, is the most commonly used technique. For example in directed diffusion [3], one of the wellknown query-based routing protocols in wireless sensor networks, a sink node is

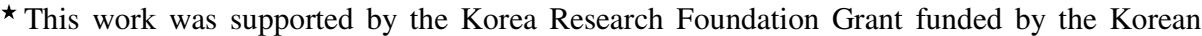
Government (R05-2003-000-10607-02004) and also supported by the MIC (Ministry of Information and Communication), Korea, under the ITRC program. 
supposed to initiate an interest packet dissemination throughout the entire network by flooding. Then a node receiving the interest sets up a gradient which indicates from whom this interest message has previously been forwarded. Although some additional feature such as a gradient reinforcement has been proposed, the directed diffusion with such a flooding of interest messages obviously increases network traffic and leads inefficient energy consumption on sensor nodes.

A geographical propagation can be more useful than flooding when an interest message needs to send towards a subset of sensor need within a certain region. Thus, a geocasting can reduce the number of redundant packets by aid of the location information of nodes. GEAR [4] is one of geocasting protocols for sensor networks. It utilizes a greedy forwarding for the packet delivery toward the target region. In greedy forwarding, a packet is forwarded to only one of the neighbor nodes whose geographical location is closest to the destination. Therefore, GEAR can minimize redundant packet traffic caused in flooding. However, most conventional geocasting protocols including GEAR consider only a single target region. The problem we are addressing in this paper is how to efficiently deliver interest messages to multiple target regions so that the latency as well as the bandwidth consumption can be reduced.

There are many sensor network applications, which require to collect the identical information from multiple regions. For example, a monitoring system in hostile environments may need to send the same advertisement to the sensors in the several regions for changing the sensing mode or interval. It may also need to send some queries such as "what's the average temperature in each target region A, B and C?". Additionally, in the battle field, the command center can give the same query to the sensors within multiple combat areas. Example queries for such scenarios would be, "How many tanks or soldiers are observed in regions X, Y and Z?" or "Where are tanks in regions X, Y and Z?". For these applications, conventional protocols need to send the identical interests to each target region multiple times. It causes significant performance degradation by increasing network traffic and wasting the energy.

We propose a noble scheme that sends interest just once at the sink node instead of multiple packet transmissions toward different target regions. Our scheme, named FERMA, creates a suitable shared path among multiple target regions. The interest messages are then forwarded along this path from the sink node to each target region through an optimal junction point. To find such an optimal junction point, we utilize the theorem of the "Fermat Point" [5]. After the interest reaches any one node in each target region, local flooding starts inside the region. A gradient, which represents the reverse path of the interest, is set up toward the sink node in the process of the interest forwarding. This gradient is utilized for actual data deliveries from sensor nodes to the sink node.

Our scheme also performs a data aggregation to reduce the amount of data traffic. It is done at each Fermat Point and entrance nodes of local flooding inside the target regions. The rest of the paper is organized as follows. Section 2 introduces the theorem of Fermat Point and its proof. In Section 3, we examine our proposed scheme in aspects of interest forwarding and data forwarding followed by ns-2 simulation results in Section 4. We conclude in Section 5. 


\section{Background and Motivation}

\subsection{Definition of the Fermat Point}

First of all, we need to explain the theorem of Fermat Point which is important to understand our scheme. Fermat point is the solution for the following question: what is the point such that the sum of its distances from the vertices of a triangle is a minimum? The definition of Fermat Point with a proof is following.

Definition. In any triangle $\triangle A B C$, we can draw three equilateral triangles $\triangle A^{\prime} B C$, $\triangle B^{\prime} C A$, and $\triangle C^{\prime} A B$ at the edges of $\triangle A B C$ as shown in Fig. 1(a). It is denoted by Fermat Point, an intersection point of three straight lines, $\overline{A A^{\prime}}, \overline{B B^{\prime}}$ and $\overline{C C^{\prime}}$, each of which connects a vertex of the given triangle and a vertex of the opposite equilateral triangle.

Theorem. Fermat Point is the point such that the sum of its distances from the vertices of a triangle is a minimum.

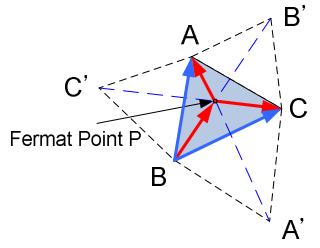

(a)

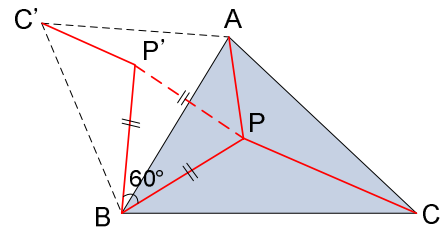

(b)

Fig. 1. The definition of Fermat Point and its proof

Proof. In $\triangle A B C$ in Fig. 1(b), select a point $P$ and connect it with vertices $A, B$, and $C$. Rotate $\triangle A B P 60^{\circ}$ around $B$ into position $\triangle C^{\prime} B P^{\prime}$. By construction, $\triangle B P P^{\prime}$ is equilateral, $\overline{P A}=\overline{C^{\prime} P^{\prime}}$, and $\overline{P B}=\overline{P^{\prime} B}$. Thus, we have $\overline{P A}+\overline{P B}+\overline{P C}=\overline{C^{\prime} P^{\prime}}+\overline{P^{\prime} P}+\overline{P C}$. As the image of $A$ under the rotation, position of $C^{\prime}$ does not depend on $P$. Also, $\overline{P A}+\overline{P B}+\overline{P C} \geq \overline{C C^{\prime}}$ because the broken line $\overline{C P P^{\prime} C^{\prime}}$ is no shorter than the straight line $\overline{C C^{\prime}}$. Therefore, $\overline{P A}+\overline{P B}+\overline{P C}$ reaches its minimum if $P$ lies on $\overline{C C^{\prime}}$. For this $P, \triangle A B C^{\prime}$ is also equilateral because $\overline{A B}=\overline{C^{\prime} B}$ and $\angle A B C^{\prime}=60^{\circ}$. With similar methods, we can draw other straight lines which connect vertices of the triangle with the opposite vertices of equilateral triangles. These straight lines cross at one point. From the definition, this point is Fermat Point. Thus, Fermat Point is the point such that the sum of its distances from the vertices of a triangle is a minimum.

The construction of Fermat Point fails if one of the internal angles of $\triangle A B C$ is $120^{\circ}$ or more. Because Fermat Point is drawn outside of the triangle. In this case, the vertex itself having the largest angle becomes an optimal point to reach each vertices of that triangle. 


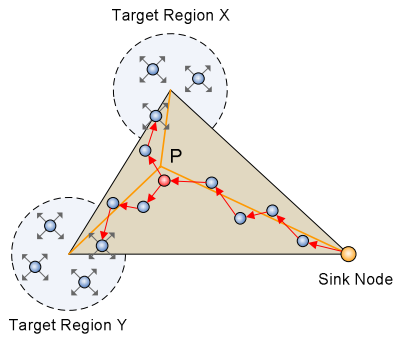

Fig. 2. Fermat Point applied in Sensor Networks with multiple target regions

\subsection{Applying Fermat Point Concept for Sensor Network Geocasting}

We illustrate how to apply the theorem of Fermat Point in wireless sensor networks. Suppose that the two points A and B of the given triangle in previous Fig. 1(b) are the two central positions of the target regions $\mathrm{X}$ and $\mathrm{Y}$, and then the point $\mathrm{C}$ is assumed to be the sink node. In this environment, the virtual triangle with three points is still formed as in Fig. 2. Therefore, we can calculate the Fermat point P, and a certain node which is closest to the Fermat Point can play a role as the junction point toward two target regions. From the sink node to this junction node, interest messages are delivered through the shared path, and then they are separated to each target region respectively. Thereby, we can optimize the interest forwarding process.

The above technique can be generalized to any number of increasing target regions as shown in Fig. 3. It may be possible to construct only one path curved severely like a circle when many target regions are placed in around the sink node. In this case, that path is extremely skewed and too long, so using such a path result in inefficiency. To solve this problem, we divide target regions into three different groups according to the angle made by them and the sink node. In Fig. 3(a), the target regions 1,2, and 3 belong to the first group, since the angles made by the sink node and these regions do not exceed 120 degrees. In Fig. 3(b), and (c), the regions 4, 5 belong to the second group, since the angles made by the sink node and these regions are in between 120 degrees and 240 degrees. In the same way, region 6 belongs to the third group. The three shared paths are set up with target regions in each group respectively. Consequently, the interest messages initiated by sink node are sent along to each path.

\section{Proposed Scheme: FERMA}

Our proposed scheme consists of two phases: interest forwarding phase and data forwarding phase. To disseminate interest messages toward multiple regions, a sink first creates a shared path based on the theorem of Fermat Point. Then, according to this path, interest messages are delivered to each target region. Any node receiving interest messages simultaneously sets up the corresponding gradient toward the previous sender for data forwarding in the next stage. More details will follow. 


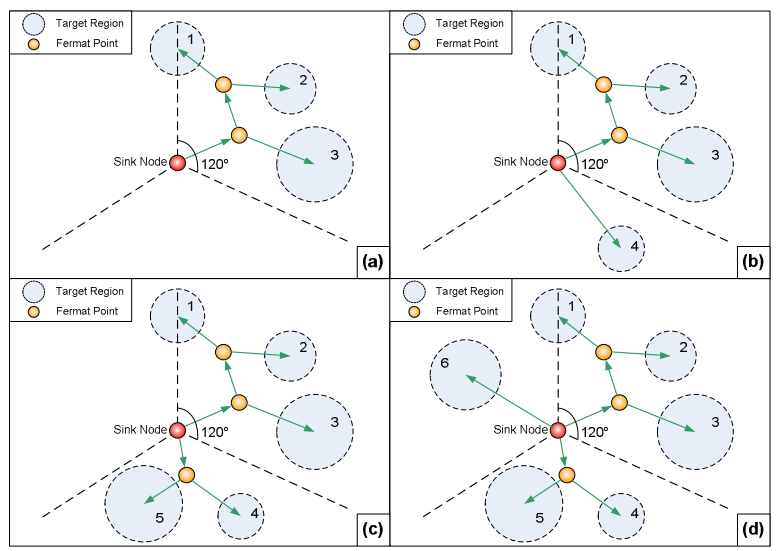

Fig. 3. Construction of the shared path in 360 degrees

\subsection{Interest Message Forwarding Phase}

For simplicity, let us consider only two target regions. Fig. 4 (a) illustrates a simple greedy forwarding approach towards the two different target regions. Since there is no optimization rule for multiple target regions in the pure greedy forwarding, the sink node is required to send an interest towards target region $\mathrm{A}$ and then, again sends the same interest message towards another target region B. When these interest packets reach in the designated target regions, local flooding is triggered, which means that all nodes within the target regions rebroadcast the receiving interests. Note that, as the number of target regions increases, the frequency of interest message transmissions by the sink also increases in this type of simple greedy approach.

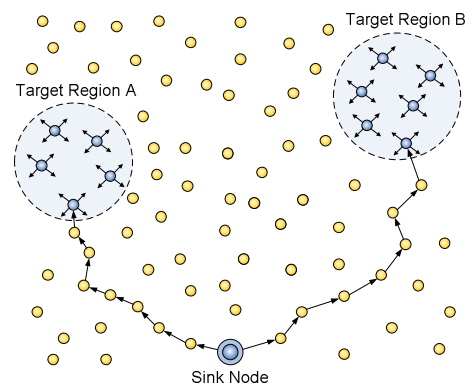

(a) Simple Greedy forwarding

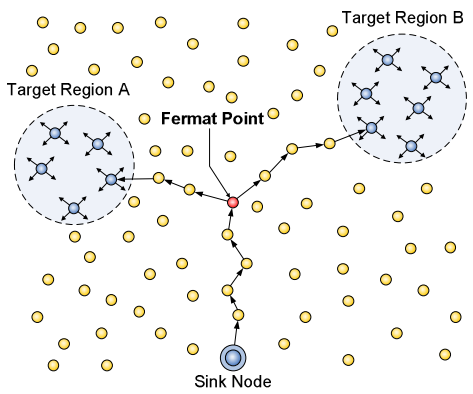

(b) The proposed FERMA forwarding

Fig. 4. Comparison of Simple Greedy protocol and FERMA protocol

On the other hand, the proposed FERMA algorithm makes a virtual triangle with three vertices including the sink node and two central points of the target regions as mentioned in the previous section. From the definition of the Fermat Point theorem, that point becomes the optimal point that minimizes the sum of distances from the 
vertices of a given triangle. It is clear that the shared path with the Fermat point is more efficient than separated multiple greedy forwarding (Compare Fig. 4(a) and (b) below).

After constructing shared path at the sink node, the interest packet is generated embedding three geographical coordinates with Fermat point and two central points of the target regions. The sink node sends this packet towards the Fermat point as the destination. Upon receiving the interest packet, each node selects the closest node to the destination as next hop among its neighbors. (For load balancing, when the node selects next hop, it can consider the amount of energy of neighbors as well as the distance to the destination. The residual energy of each neighbor can be informed by hello message) When a node could not find its neighbor node which is closer to the destined Fermat point, it becomes Fermat point by itself. Through this mechanism, the interest forwarding to Fermat point never fails because geographically closest node is always selected as the junction point. The next step is splitting of interests toward two target regions. Separated interest packets are forwarded to each target region respectively. Finally, when the interest reached any node inside the target region, local flooding is started. Local flooding means these flooded packets do not leak out of the target region. If any node located outside of the target region receives these locally flooded packets, it simply drops the packets.

Let us consider a generalization in more than two target regions. The basic idea is to chain consecutive Fermat points. Fig. 5 illustrates three target regions. Here, we calculate the first Fermat point ' $F 1$ ' from the virtual triangle formed with the two target regions $\mathrm{A}, \mathrm{B}$ and the sink. Next, the second Fermat point ' $\mathrm{F} 2$ ' is obtained with the first Fermat point $\mathrm{F} 1$, the target region $\mathrm{C}$ and the sink node. In this way, we can combine additional target regions into the previously constructed path. Ultimately, our scheme is scalable with the large number of target regions as mentioned in section 2.2.

After calculating the path, the sink node sends the interest towards the second Fermat point F2 with the intermediate location list including F2, C, F1, B, and A as seen in Fig. 5. The intermediate location list is filled with all locations that this packet goes through including the Fermat points and target regions. When this packet reaches F2, it is split into two packets, and then one is transmitted to the first Fermat point F1 with reduced intermediate location list including F1, B, A, and another is transmitted to the target region $\mathrm{C}$. Arriving at F1, this packet is also separated into two packets for the target regions $\mathrm{A}$ and $\mathrm{B}$.

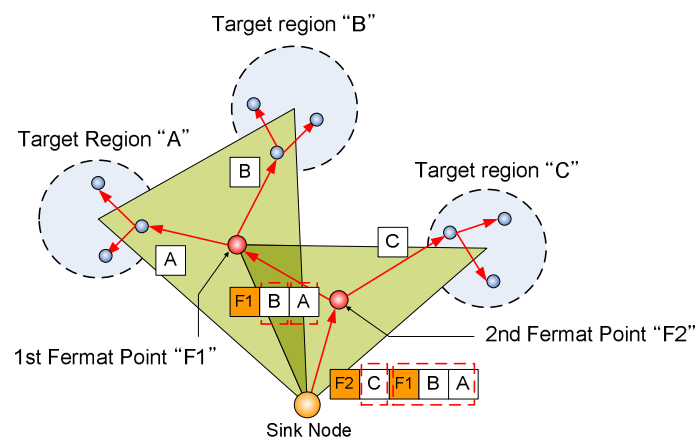

Fig. 5. Interest forwarding along the shared path 


\subsection{Data Packet Forwarding Phase}

In the process of the interest packet forwarding, all nodes record the previous hop node which forwards that interest. It is called gradient, and used in several sensor network protocols, for example directed diffusion. If any node in the target region has data matched with the condition recorded in the interest packets, it sends these data to the sink node through the reverse path according to the gradient. Every intermediate node keeps records of the gradient, so packet can be delivery up to the sink node.

Some kind of data aggregation, for example the suppression of duplication, does not need any specific points to collect data packets. It is simply performed in any nodes using data cache. On the other hand, there are other aggregation functions as minima, maxima, average, etc. These functions are able to produce more exact and reliable results when a number of sensor nodes collaborate together. In this case, some intermediate nodes are required to collect data packets and to execute aggregation functions. Generally, in the cluster head or the intersection between multi-paths, the data aggregation is performed.

In our scheme, data aggregation is achieved as two levels. The first level aggregation is performed within each target region. In Fig. 6(a), the node L1, which is called to the first level aggregator, receives data packets A1 and A2 from the same region. After a while, L1 transmits the aggregated packet AA towards the sink node. This function is also executed in L2 and L3 in other target regions. Actually, these first level aggregators L1, L2 and L3 are the initial nodes starting the local flooding in the interest forwarding. These preliminary aggregated packets AA, BB and CC are aggregated ever further when they reach the Fermat points. Thus, F1 node aggregates the packets $\mathrm{AA}$ and $\mathrm{BB}$, and also this aggregated packet is aggregated once more in the second level aggregator $\mathrm{F} 2$. These second level aggregator are exactly identical to the Fermat point nodes.

The data aggregation techniques adapted in our scheme does not require can reduce the total number of data packets without requiring any additional control packets. However, the latency of data delivery becomes a litter longer, because it needs to wait for the data packets during a certain time. The latency and the energy efficiency are trade-off. Therefore, in such a sensor network which requires more energy conservation than latency, our data aggregation techniques are definitely helpful.

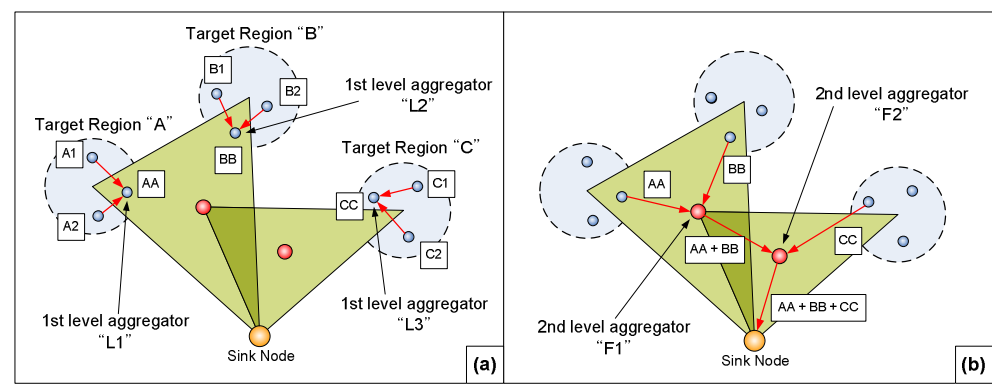

(a) Data aggregation in each target region

(b) Data aggregation at Fermat point

Fig. 6. Two-level data aggregation 


\section{Performance Evaluation}

In this section, we evaluate the performance of our scheme. Our primary performance metrics are the packet overhead and the energy consumption decreased due to path optimization toward multiple regions. Moreover, we are interest in how the performance of our scheme is affected by the variation of the number of target region.

In our simulation, the proposed scheme is compared to pure greedy forwarding and the flooding. We modify them, since they have no consideration of multiple target regions in interest delivery. In addition, they should be able to deliver the data packet from source node to sink node. Thus, we have augmented pure greedy forwarding with the multiple transmissions of interest packet toward each target region, local flooding within the target regions and data delivery through the gradient setup. In the flooding used in our simulation, the interest packet contains the location coordinates of all target regions. Thereby, the interest packet can is forwarded just once for multiple target regions. However, this method is still less efficient. The data forwarding is achieved through the flooding likewise interest forwarding.

\subsection{Simulation Environment}

We preformed a simulation using $n s-2$. In our simulation model, 100 nodes are randomly placed in $200 m \times 200 m$ square area. The transmission range of each node is $40 \mathrm{~m}$. We consider the immobile sensor network, so every sensor node is static. We locate one sink node at coordinate $(0,0)$ for convenience. The number of target regions varies from 1 to 5 , and the target region is circularly shaped with the radius of $25 \mathrm{~m}$. The interest packet with fixed size payload of 36 bytes is periodically generated every 5 seconds from the sink node. In order to respond to the interests, the source node sends the data packet with fixed size payload of 64 bytes each. Total simulation time is 500 seconds and we repeat each scenario ten times with different randomly deployed nodes. We choose the following two metrics to analyze the performance of our scheme:

Average Packet Overhead. We measure the number of packets divided by the total number of nodes and the total number of queries generated from the sink node. The total number of queries is obtained by the simulation time divided by the interval of query generation. This metric reflects the overhead of packet transmissions by each node to deliver a query. We also examine the packet overhead on the aspect to packet size. It means that the total size of transmitted packets divided by the total number of nodes and the total number of queries generated from sink node.

Average Consumed Energy. We measure the total energy consumption of all nodes divided by the total number of nodes and the total number of queries in the same manner as the average packet overhead.

\subsection{Simulation Results}

The first evaluation is about the average packet overhead as a function of the number of target region. Fig. 8(a) shows the average interest packet overhead and Fig. 8(b) shows the average data packet overhead. In Fig. 8(a), when the interest is disseminated through flooding, we observe that every node transmits one interest packet per a query all the time. It means that every node has to attend to the interest 
dissemination. Thus, flooding is the most inefficient method among three protocols that we examine. Our FERMA generates fewer interest packets than the greedy forwarding as much as maximum $29 \%$. The difference of amount of interest packets is getting larger as the number of target region increases. It means that FERMA improve the performance much more as the number of source nodes increases. Such an improvement is achieved as our scheme uses the optimal shared path for multiple target regions. In addition, Fig. 8(b) shows FERMA forwards data packets more efficiently than the other two protocols. The two-level data aggregation enables the data packets from different source nodes to be collected and merged. Thereby, our scheme reduces a lot of the network traffic. Especially in the best case, our scheme produces fewer packets up to $1 / 15$ of flooding, and $1 / 3$ of greedy forwarding.

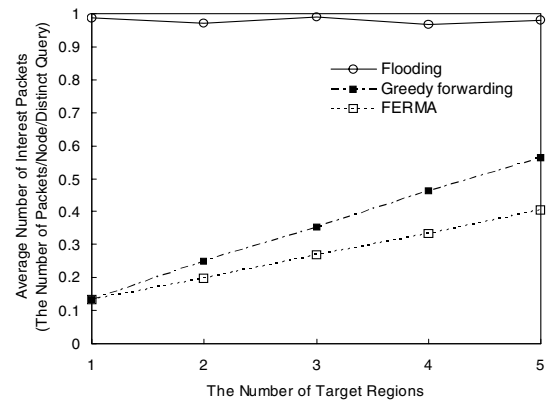

(a)

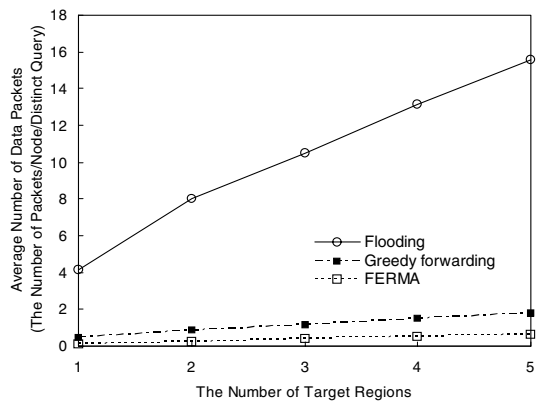

(b)

Fig. 8. Interest Packet Overhead and Data Packet Overhead

Fig. 9 shows the result of packet overhead on the aspect of the size of total packets including interest, data and hello messages. As seen in this figure, FERMA is more efficient than greedy forwarding as much as maximum 57\%. Fig 10 represents the average consumed energy in three protocols. This figure shows that our scheme make the network nodes consume energy resources more efficiently than any others. Consequently, we can extend the life time of the network with the proposed scheme.

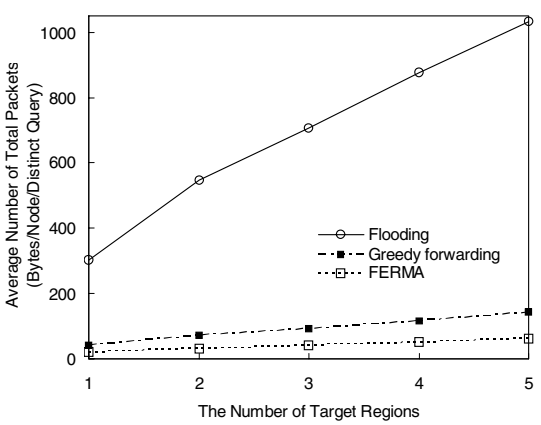

Fig. 9. Total Packet Overhead

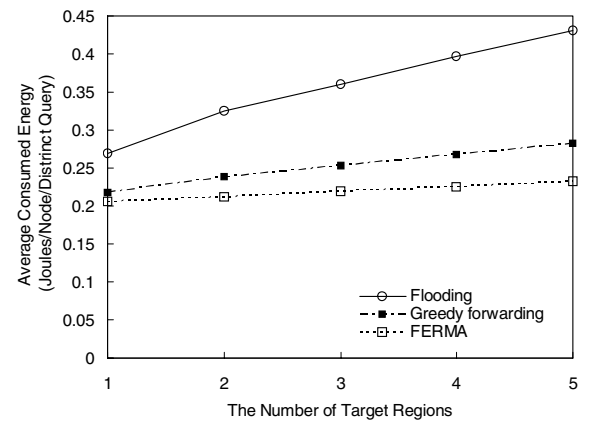

Fig. 10. Energy Consumption 


\section{Conclusion}

In this paper, we focus on the efficient interest forwarding in the environment of multiple target regions. We propose a noble scheme which sends an interest message from the sink node at once instead of multiple packet transmissions towards different target regions respectively. The proposed FERMA makes a suitable shared path among multiple target regions using the theorem of Fermat Point. In addition, we examine the two-level data aggregation scheme to reduce more data packet overhead. Consequently, our scheme optimizes the delivery of interest messages and replied data. We prove such an improvement in terms of packet overhead and energy consumption through simulation studies. FERMA is useful in many applications and scenarios to desire interest dissemination frequently towards multiple target regions. Ongoing work includes avoiding the obstacle and exploring other situation in which performance degradation may occur.

\section{References}

1. I.F. Akyildiz, W. Su, Y. Sankarasubramaniam and E. Cayirci, "Wireless Sensor Networks : A Survey”, Computer Networks, vol.38, 2002, pp. 392-422.

2. J. N. Al-Karaki and A. E. Kamal, "Routing Techniques in Wireless Sensor Networks: A Survey", IEEE Wireless Communications, vol. 11(6), 2004, pp 6-28.

3. C. Intanagonwiwat, R. Govindan, D. Estrin and J. Heidemann, "Directed Diffusion for Wireless Sensor Networking”, IEEE/ACM Transactions on Networking, vol. 11(1), 2003, pp. 2-16.

4. Y. Yu, R. Govindan and D. Estrin, "Geographical and Energy Aware Routing: A Recursive Data Dissemination Protocol for Wireless Sensor Networks", UCLA Comp. Sci. Dept. tech. rep., UCLA-CSD TR-010023, 2001.

5. The Fermat Point and Generalizations. [Online]. Available: http://www.cut-the-knot.org/ Generalization/fermat_point.shtml 